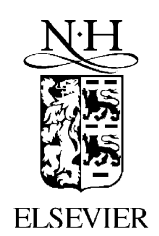

Mathematics and Computers in Simulation 47 (1998) 329-346

\title{
Nonlocal Monte Carlo algorithms for statistical physics applications
}

\author{
Wolfhard Janke ${ }^{1}$ \\ Institut für Physik, Johannes Gutenberg-Universität, D-55099 Mainz, Germany
}

\begin{abstract}
After a brief general overview of Monte Carlo computer simulations in statistical physics, special emphasis is placed on applications to phase transitions and critical phenomena. Here, standard simulations employing local update algorithms are severely hampered by the problem of critical slowing down, that is by strong correlations between successively generated data. It is shown that this problem can be greatly reduced by using nonlocal update techniques such as cluster and multigrid algorithms. The general ideas are illustrated for simple lattice spin models and Euclidean path integrals. (C) 1998 IMACS/ Elsevier Science B.V.
\end{abstract}

Keywords: Monte Carlo simulations; Importance sampling; Cluster algorithms; Multigrid techniques; Phase transitions; Critical phenomena

\section{Introduction}

Statistical physics of complex systems pose many hard problems which can often be treated only approximately by analytical approaches. It is therefore important to complement such studies by numerical computer simulations. The applications range from systems like (spin) glasses and disordered magnets to polymers or proteins, to mention only a few classical problems. Quantum statistical problems in condensed-matter physics or the broad field of elementary particles and quantum gravity in high-energy physics are examples of another important class of applications.

The numerical tools can roughly be divided into molecular dynamics (MD) and Monte Carlo (MC) simulations. In the past few years the predictive power of the MC approach in particular was considerably enhanced by the development of nonlocal update algorithms. Not all of them are already well-enough understood to be applicable to really complex physical systems, but it is encouraging that at least for relatively simple systems orders of magnitude of computing time can be saved by these refinements.

\footnotetext{
${ }^{1}$ Tel.: +49 6131 393641; +49 6131 395441; e-mail: janke@miro.physik.uni-mainz.de
} 
The general ideas underlying these algorithms will be illustrated for classical $\mathrm{O}(n)$ symmetric spin systems such as Ising $(n=1), \mathrm{XY}(n=2)$, and Heisenberg $(n=3)$ models, and for simple quantum statistical problems in the path-integral representation. In the first case this allows comparisons with completely different theoretical approaches such as field theory or series expansions, and, of course, with experiments. And in the second example one can sometimes compare with numerical solutions of the associated Schrödinger equation.

The rest of these notes is organized as follows. In Section 2, I first summarize some properties of phase transitions and critical phenomena, and recall the definition of $\mathrm{O}(n)$ spin models. In Section 3, the background of MC simulations in statistical physics and the standard Metropolis update algorithm are described. The main material is presented in Section 4, which is devoted to an overview of nonlocal update algorithms with emphasis on cluster and multigrid techniques, and I conclude with a few final comments in Section 5.

\section{Phase diagrams and phase transitions}

In limiting cases such as low and high temperatures (or magnetic fields, pressure, etc.) the physical degrees of freedom usually decouple and the statistical mechanics of even complex systems become quite manageable, both analytically and numerically. Much more interesting is the region in between these extremes where strong cooperation effects may cause phase transitions, e.g., from an ordered phase at low temperatures to a disordered phase at high temperatures. An example is the melting line in the phase diagram of matter sketched on the 1.h.s. of Fig. 1. The prediction of the physical properties of this most difficult region of a phase diagram as accurately as possible is the most challenging objective of all statistical mechanics approaches, including numerical simulations.

The theory of phase transitions is a very broad subject described comprehensively in many textbooks (for an introduction see, e.g., [1]). Here we shall be content with a rough classification into first-order and second-order (or, more generally, continuous) phase transitions, and a summary of those properties that are most relevant for numerical simulations. Some characteristic properties of first- and secondorder phase transitions are sketched in Fig. 2.

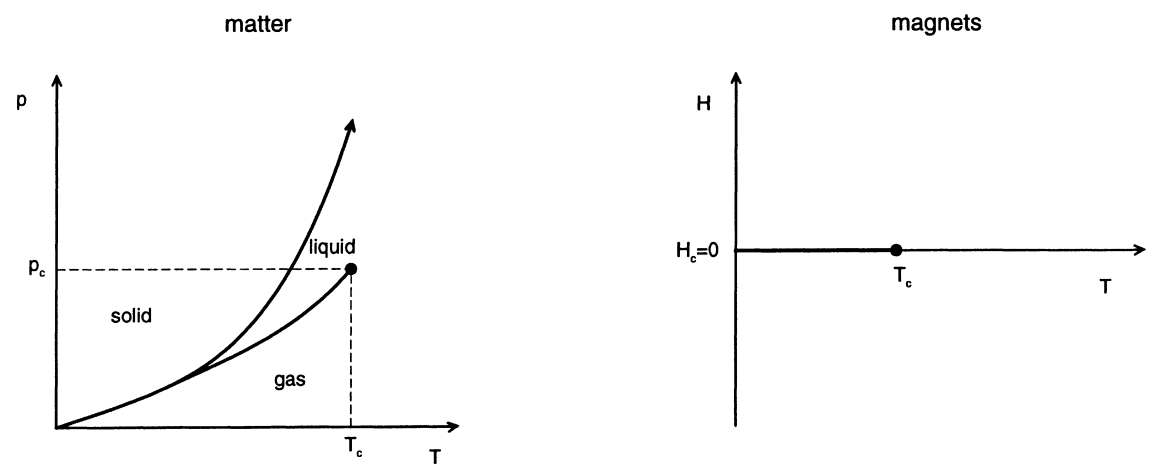

Fig. 1. The phase diagrams of matter in the temperature-pressure plane and of magnets in the temperature-field plane. The solid lines are first-order phase transitions, and the filled circles indicate critical points. 

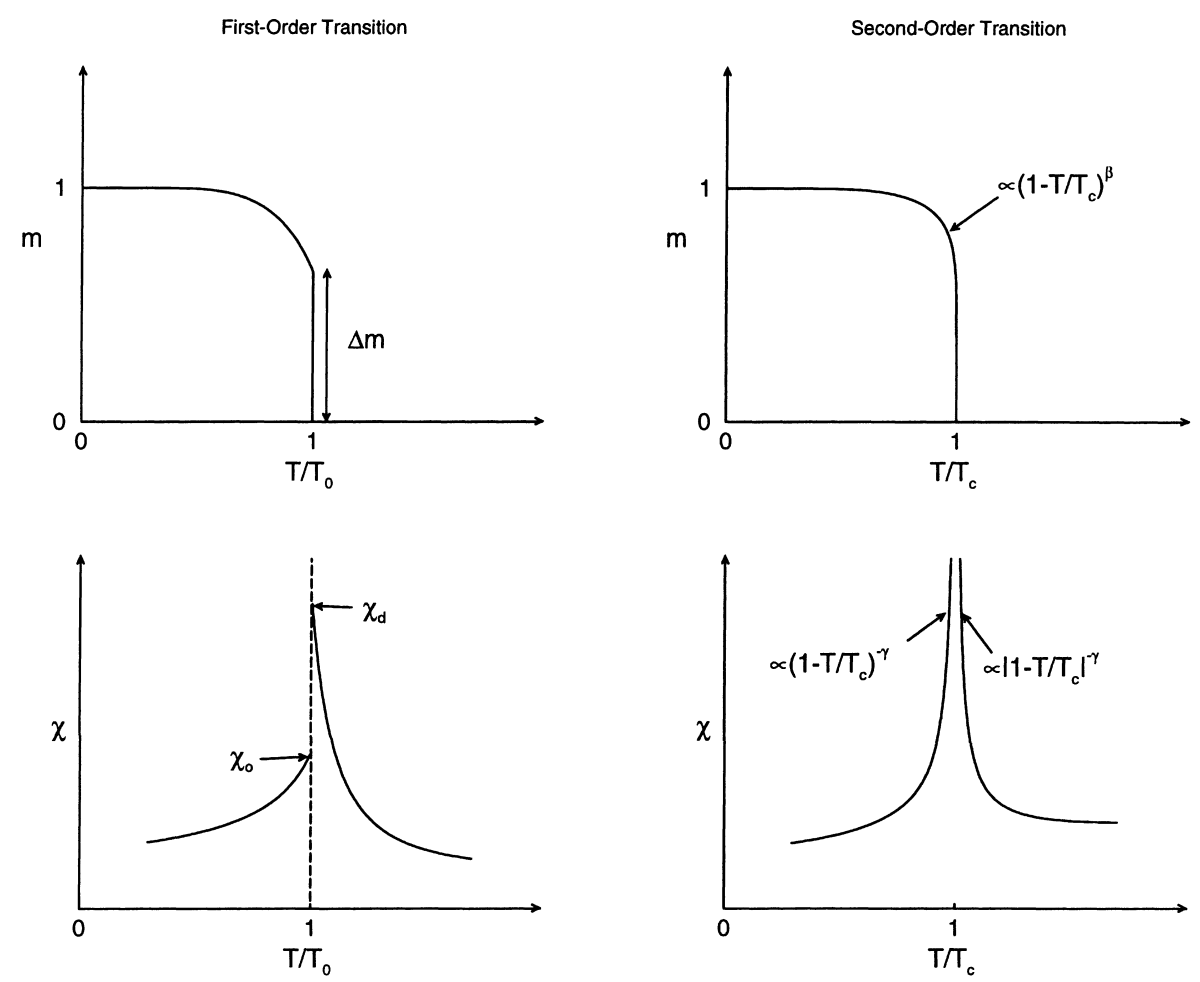

Fig. 2. The characteristic behavior of the magnetization, $m$, and susceptibility, $\chi$, at first- and second-order phase transitions.

\subsection{First-order phase transitions}

Most phase transitions in nature are of first order [2-5]. The best-known example sketched on the r.h.s. of Fig. 1 is the field-driven transition in magnets at temperatures below the Curie point. The paradigm of a temperature-driven first-order transition experienced every day is ordinary melting [6,7].

In general, first-order phase transitions are characterized by discontinuities of the order parameter (the jump $\Delta m$ of the magnetization, $m$, in Fig. 2), or the energy (the latent heat $\Delta e$ ), or both. This reflects the fact that, at the transition temperature $T_{0}$, two (or more) phases can coexist. In the example of a magnet at low temperatures the coexisting phases are the phases with positive and negative magnetization, whereas at the melting transition they are the solid (ordered) and liquid (disordered) phases. The correlation length in the coexisting pure phases is finite. Consequently the specific heat, $C$, and the susceptibility, $\chi$, in the pure phases stay finite at the transition point. Mathematically there are, however, superimposed delta function-like singularities associated with the jumps of $e$ and $m$.

\subsection{Second-order phase transitions}

In this paper we will mainly consider second-order phase transitions and critical phenomena [1], which are characterized by a divergent correlation length $\xi$ at the transition temperature $T_{\mathrm{c}} \equiv 1 / \beta_{\mathrm{c}}$. This is illustrated in Fig. 3, where two typical configurations of the 2D Ising model at $T \gg T_{\mathrm{c}}$ and 

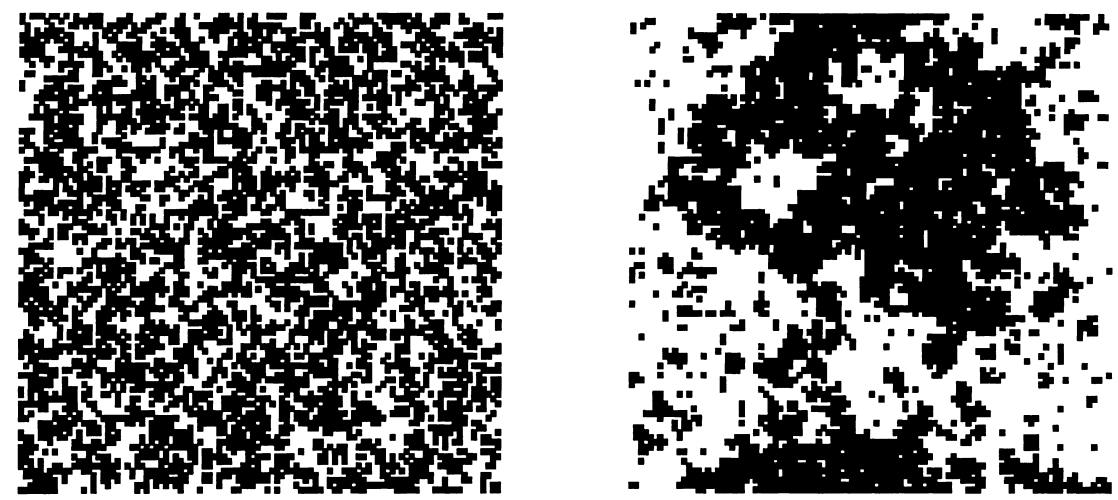

Fig. 3. Typical 2D Ising model configurations on a $100 \times 100$ lattice at $\beta / \beta_{\mathrm{c}}=0.50$ (left) and 0.98 (right).

$T \approx T_{\mathrm{c}}$ are shown. Because for an infinite correlation length fluctuations on all length scales are equally important, one expects $T_{\mathrm{c}}$ power-law singularities in thermodynamic functions. The leading singularity of the correlation length is usually parametrized in the high-temperature phase as

$$
\xi=\xi_{0^{+}}\left|1-T / T_{\mathrm{c}}\right|^{-\nu}+\cdots \quad\left(T \geq T_{\mathrm{c}}\right)
$$

where the.. indicate subleading corrections (analytical as well as confluent). This defines the critical exponent $\nu$ and the critical amplitude $\xi_{0^{+}}$on the high-temperature side of the transition. In the lowtemperature phase one expects a similar behavior with the same critical exponent $\nu$ but a different critical amplitude $\xi_{0^{-}} \neq \xi_{0^{+}}$. The singularities of the specific heat, magnetization, and susceptibility are similarly parametrized by the critical exponents $\alpha, \beta$, and $\gamma$, respectively:

$$
\begin{aligned}
& C=C_{\text {reg }}+C_{0}\left|1-T / T_{\mathrm{c}}\right|^{-\alpha}+\cdots \\
& m=m_{0}\left(1-T / T_{\mathrm{c}}\right)^{\beta}+\cdots \\
& \chi=\chi_{0}\left|1-T / T_{\mathrm{c}}\right|^{-\gamma}+\cdots
\end{aligned}
$$

where $C_{\text {reg }}$ is a regular background term, and the amplitudes are again different on the two sides of the transition (cf. Fig. 2).

An important feature of second-order phase transitions is that due to the divergence of $\xi$ the shortdistance details of the Hamiltonian should not matter. This is the basis of the universality hypothesis which states that all systems with the same symmetries and same dimensionality should exhibit similar singularities governed by one and the same set of critical exponents. For the amplitudes this is not true, but certain amplitude ratios are also universal.

For systems of finite size, as in any numerical simulation, the correlation length cannot diverge, and the divergencies in all other quantities are also rounded and shifted. Near $T_{\mathrm{c}}$ the role of $\xi$ in the scaling formulas is then taken over by the linear size of the system, $L$. By writing $\left|1-T / T_{\mathrm{c}}\right| \propto \xi^{-1 / \nu} \rightarrow L^{-1 / \nu}$, we see that at $T_{\mathrm{c}}$ the scaling laws (2)-(4) are replaced by the finite-size scaling (FSS) ansätze,

$$
C=C_{\text {reg }}+a L^{\alpha / \nu}+\cdots
$$




$$
\begin{aligned}
& m \propto L^{-\beta / \nu}+\cdots \\
& \chi \propto L^{\gamma / \nu}+\cdots
\end{aligned}
$$

More generally these scaling laws are valid in the vicinity of $T_{\mathrm{c}}$ as long as the scaling variable $x=\left(1-T / T_{\mathrm{c}}\right) L^{1 / \nu}$ is kept fixed [8-11]. In particular this is true for the locations $T_{\max }$ of the (finite) maxima of thermodynamic quantities, which are expected to scale with the system size as $T_{\max }=T_{\mathrm{c}}\left(1-x_{\max } L^{-1 / \nu}+\cdots\right)$. In this more general formulation the scaling law for the susceptibility, e.g., reads $\chi(T, L)=L^{\gamma / \nu} f_{\chi}(x)+\cdots$.

\subsection{Spin models}

In the following we shall illustrate the numerical techniques used to study phase transitions by $\mathrm{O}(n)$ symmetric spin models whose canonical partition function is defined as

$$
Z_{n}(\beta)=\sum_{\left\{\vec{\sigma}_{i}\right\}} \exp \left(-\beta H_{n}\right)
$$

with

$$
H_{n}=-J \sum_{\langle i j\rangle} \vec{\sigma}_{i} \cdot \vec{\sigma}_{j}, \quad \vec{\sigma}_{i}=\left(\sigma_{i}^{(1)}, \sigma_{i}^{(2)}, \ldots, \sigma_{i}^{(n)}\right),\left|\vec{\sigma}_{i}\right|=1
$$

Here $\beta=1 / k_{B} T$ is the inverse temperature, the spins $\vec{\sigma}_{i}$ live on the sites $i$ of a $D$-dimensional cubic lattice of volume $V=L^{D}$, and the symbol $\langle i j\rangle$ indicates that the lattice sum runs over all DV nearestneighbor pairs. We always assume periodic boundary conditions. For $n=1$ this reduces to the wellknown Ising model [12],

$$
H_{1}=-J \sum_{\langle i j\rangle} \sigma_{i} \sigma_{j} \quad \sigma_{i}= \pm 1 \text { (Ising) }
$$

which is presumably the best studied lattice model in statistical physics.

Standard observables are the internal energy per site, $e=E / V$, with $E=-\mathrm{d} \ln Z_{n} / \mathrm{d} \beta \equiv\left\langle H_{n}\right\rangle$, and the specific heat per site, $C=\mathrm{d} e / \mathrm{d} T=k_{B} \beta^{2}\left(\left\langle H_{n}^{2}\right\rangle-\left\langle H_{n}\right\rangle^{2}\right) / V$. On finite lattices the magnetization and susceptibility are usually defined as $m=M / V=\left\langle\left|\vec{\sigma}_{\mathrm{av}}\right|\right\rangle$ with $\vec{\sigma}_{\mathrm{av}}=\sum_{i} \vec{\sigma}_{i} / V$, and $\chi=\beta V\left(\left\langle\vec{\sigma}_{\mathrm{av}}^{2}\right\rangle\right.$ $\left.-\left\langle\left|\vec{\sigma}_{\mathrm{av}}\right|\right\rangle^{2}\right)$. In the high-temperature phase one often employs the fact that the magnetization vanishes in the infinite volume limit and defines $\chi^{\prime}=\beta V\left\langle\vec{\sigma}_{\text {av }}^{2}\right\rangle$. Similarly, the correlation length $\xi=$ $-\lim _{|\vec{x}| \rightarrow \infty}|\vec{x}| / \ln G(\vec{x})$ can be extracted from the exponential decay at large distances of the spin-spin correlation function $G\left(\vec{x}_{i}-\vec{x}_{j}\right)=\left\langle\vec{\sigma}_{i} \cdot \vec{\sigma}_{j}\right\rangle$.

\section{The Monte Carlo method}

Even for the simplest model, the Ising model with only two possible states per site, a direct summation of the partition function is impossible for any reasonable lattice size. For a still quite modest 2 D $50 \times 50$ system we would have to handle an enormous number of terms: $2^{2500} \approx 10^{753}$. It 
goes without saying that here even a grand-challenge Terraflop computer would not be of any help.

A naive random MC sampling of the spin configurations also does not work, basically because of the huge number of degrees of freedom which distinguishes the statistical physics applications from the treatment of ordinary many-dimensional integrals. The problem is that the relevant region in the highdimensional phase space is extremely narrow and hence too rarely hit by random sampling. To appreciate this problem it is helpful to realize that random sampling corresponds to a simulation at $\beta=0$, i.e., at infinite temperature. The interesting physics, however, usually takes place at finite temperatures $T \ll \infty$, and information about this temperature region is only coded in the exponentially suppressed tails of randomly sampled distributions.

The solution to this problem has been known for a long time. One has to use the importance sampling technique [13] which is designed to draw configurations according to their Boltzmann weight,

$$
P^{\mathrm{eq}}\left[\left\{\sigma_{i}\right\}\right] \propto \exp \left(-\beta H\left[\left\{\sigma_{i}\right\}\right]\right)
$$

In more mathematical terms one sets up a Markov chain,

$$
\ldots \stackrel{W}{\rightarrow}\left\{\sigma_{i}\right\} \stackrel{W}{\rightarrow}\left\{\sigma_{i}^{\prime}\right\} \stackrel{W}{\rightarrow}\left\{\sigma_{i}^{\prime \prime}\right\} \stackrel{W}{\rightarrow} \ldots
$$

with a transition operator $W$ satisfying the conditions

$$
\begin{array}{ll}
\text { (a) } & W\left(\left\{\sigma_{i}\right\} \rightarrow\left\{\sigma_{i}^{\prime}\right\}\right)>0, \quad \text { for all }\left\{\sigma_{i}\right\},\left\{\sigma_{i}^{\prime}\right\} \\
\text { (b) } & \sum_{\left\{\sigma_{i}^{\prime}\right\}} W\left(\left\{\sigma_{i}\right\} \rightarrow\left\{\sigma_{i}^{\prime}\right\}\right)=1 \text { for all }\left\{\sigma_{i}\right\} \\
\text { (c) } & \sum_{\{\sigma\}_{i}} W\left(\left\{\sigma_{i}\right\} \rightarrow\left\{\sigma_{i}^{\prime}\right\}\right) P^{\mathrm{eq}}\left[\left\{\sigma_{i}\right\}\right]=P^{\mathrm{eq}}\left[\left\{\sigma_{i}^{\prime}\right\}\right] \text { for all }\left\{\sigma_{i}^{\prime}\right\}
\end{array}
$$

From Eq. (14) we see that $P^{\mathrm{eq}}$ is a fixed point of $W$. A somewhat simpler sufficient condition is detailed balance:

$$
P^{\mathrm{eq}}\left[\left\{\sigma_{i}\right\}\right] W\left(\left\{\sigma_{i}\right\} \rightarrow\left\{\sigma_{i}^{\prime}\right\}\right)=P^{\mathrm{eq}}\left[\left\{\sigma_{i}^{\prime}\right\}\right] W\left(\left\{\sigma_{i}^{\prime}\right\} \rightarrow\left\{\sigma_{i}\right\}\right)
$$

After an initial equilibration time, expectation values can then be estimated as an arithmetic mean over the Markov chain, eg.,

$$
\langle H\rangle=\sum_{\left\{\sigma_{i}\right\}} H\left[\left\{\sigma_{i}\right\}\right] P^{\mathrm{eq}}\left[\left\{\sigma_{i}\right\}\right] \approx \frac{1}{N} \sum_{j=1}^{N} H\left[\left\{\sigma_{i}\right\}\right]_{j}
$$

A more detailed exposition of the basic concepts underlying any MC simulation can be found in many textbooks and reviews [8,13-16].

\subsection{Metropolis algorithm}

In the standard Metropolis algorithm [17] the Markov chain is realized by proposing locally updates of single spins and accepting them with probability 


$$
W\left(\left\{\sigma_{i}\right\} \rightarrow\left\{\sigma_{i}^{\prime}\right\}\right)= \begin{cases}\exp \left[-\beta\left(E^{\prime}-E\right)\right] & E^{\prime}>E \\ 1 & E^{\prime} \leq E\end{cases}
$$

where $E$ and $E^{\prime}$ denote the energy before and after the spin flip. If the energy is lowered, the spin flip is always accepted. But even if the energy is increased, the flip has to be accepted with a certain probability to ensure the proper treatment of entropic contributions.

There are many ways of choosing the spins to be updated. The lattice sites may be picked at random or according to a random permutation, which can be updated every now and then. But a simple fixed lexicographical order is also permissible. Or one updates first all odd and then all even sites, which is the usual choice in vectorized codes. A so-called lattice sweep is completed on average ${ }^{2}$ when an update is proposed for all spins.

The advantage of this simple algorithm is its flexibility which allows its application to a great variety of physical systems. The great disadvantage, however, is that this algorithm is plagued by strong correlations between successively generated data. This leads to large statistical errors:

$$
\epsilon=\epsilon_{\text {naive }} \sqrt{2 \tau_{\text {int }}}
$$

where $\epsilon_{\text {naive }}=\sqrt{\sigma^{2} / N}$ is the statistical error of $N$ uncorrelated measurements with variance $\sigma^{2}$, and

$$
\tau_{\text {int }}=\frac{1}{2}+\sum_{j=1}^{N} A(j)\left(1-\frac{j}{N}\right)
$$

is the so-called integrated autocorrelation time derived from the autocorrelation function [18]:

$$
A(j)=\frac{\left\langle\mathcal{O}_{i} \mathcal{O}_{i+j}\right\rangle-\left\langle\mathcal{O}_{i}\right\rangle\left\langle\mathcal{O}_{i}\right\rangle}{\left\langle\mathcal{O}_{i}^{2}\right\rangle-\left\langle\mathcal{O}_{i}\right\rangle\left\langle\mathcal{O}_{i}\right\rangle}
$$

where $\mathcal{O}_{i}$ stands generically for a quantity measured at "time" $i$.

Empirically one finds that the autocorrelation time grows proportional to the spatial correlation length,

$$
\tau \propto \xi^{z}
$$

with a dynamical critical exponent $z \approx 2$. Heuristically this can be understood by assuming that local excitations diffuse through the system like a random walk. Since $\xi$ diverges at criticality, the Metropolis algorithm thus suffers severely from critical slowing down. Of course, in finite systems $\xi$ cannot diverge. Then $\xi$ is replaced by the linear lattice size $L$, yielding $\tau \propto L^{z}$.

\section{Nonlocal Monte Carlo algorithms}

The problem of critical slowing down can be overcome by nonlocal update algorithms. In the past few years several different types of such algorithms have been proposed. Here we shall concentrate on cluster and multigrid update algorithms.

\footnotetext{
${ }^{2}$ This is relevant only in the case where the lattice sites are picked at random.
} 


\subsection{Cluster algorithms}

Let us first consider the Ising model, where the prescription for cluster update algorithms can easily be read off from the equivalent Fortuin-Kasteleyn representation [19],

$$
\begin{aligned}
& Z=\sum_{\left\{\sigma_{i}\right\}} \exp \left(\beta \sum_{\langle i j\rangle} \sigma_{i} \sigma_{j}\right) \\
& =\sum_{\left\{\sigma_{i}\right\}} \prod_{\langle i j\rangle} \mathrm{e}^{\beta}\left[(1-p)+p \delta_{\sigma_{i} \sigma_{j}}\right] \\
& =\sum_{\left\{\sigma_{i}\right\}} \sum_{\left\{n_{i j}\right\}} \prod_{\langle i j\rangle} \mathrm{e}^{\beta}\left[(1-p) \delta_{n_{i j}, 0}+p \delta_{\sigma_{i} \sigma_{j}} \delta_{n_{i j}, 1}\right]
\end{aligned}
$$

where the $n_{i j}$ are auxiliary bond variables. Only if the two spins $\sigma_{i}$ and $\sigma_{j}$ are equal, the $n_{i j}$ can be "activated" $\left(n_{i j}=1\right)$ with probability

$$
p=1-\mathrm{e}^{-2 \beta}
$$

In Eq. (23) we used the fact that the product $\sigma_{i} \sigma_{j}$ of two Ising spins can only take the two values \pm 1 , and Eq. (24) follows from the trivial identity $a+b=\sum_{n=0}^{1}\left(a \delta_{n, 0}+b \delta_{n, 1}\right)$.

\subsubsection{Swendsen-Wang cluster}

According to the joint probability distribution in Eq. (24) a cluster update sweep then consists of alternating updates of the bond variables $n_{i j}$ for given spins with updates of the spins $\sigma_{i}$ for a given bond configuration:

1. If $\sigma_{i} \neq \sigma_{j}$, set $n_{i j}=0$, or if $\sigma_{i}=\sigma_{j}$, assign values $n_{i j}=1$ and 0 with probability $p$ and $1-p$, respectively (see Fig. 4).

2. Identify clusters of spins that are connected by "active" bonds $\left(n_{i j}=1\right)$.

3. Draw a random value \pm 1 independently for each cluster (including one-site clusters), which is then assigned to all spins in a cluster.

Technically the cluster identification part is the most complicated step, but there are by now quite a few efficient algorithms available which can even be used on parallel computers [20]. Vectorization, on the other hand, is only partially possible [21].
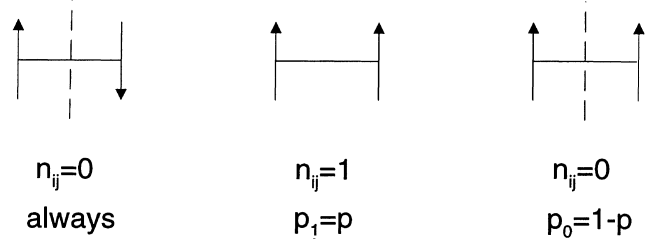

Fig. 4. Illustration of the bond variable update. A bond between like spins is "active" with probability $p=1-\exp (-2 \beta)$. 

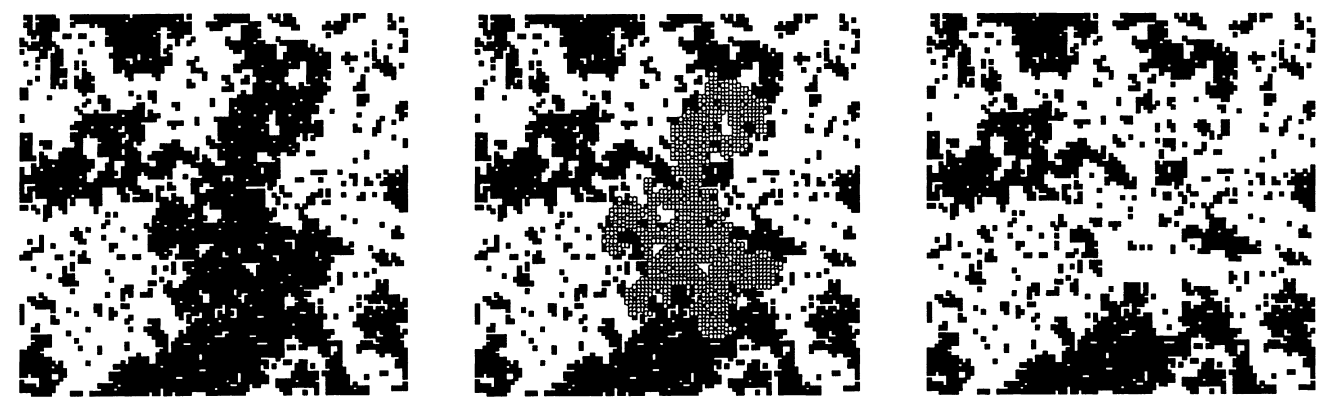

Fig. 5. Illustration of the Wolff cluster update.

Notice the difference between the just-defined stochastic clusters and geometric clusters whose boundaries are defined by drawing lines through bonds between unlike spins. Since in the stochastic definition also bonds between like spins are "deleted" with probability $p_{0}=1-p=\exp (-2 \beta)$, stochastic clusters are on average smaller than geometric clusters. Only at zero temperature $(\beta \rightarrow \infty)$ does $p_{0}$ approach zero and the two cluster definitions coincide.

As described above, the whole lattice is decomposed into stochastic clusters whose spins are assigned a random value +1 or -1 . This is the original Swendsen-Wang (SW) [22] or multiple-cluster formulation.

\subsubsection{Wolff cluster}

In the Wolff [23] or single-cluster (SC) formulation only one cluster is flipped at a time. Here one chooses a lattice site at random, constructs only the cluster connected with this site, and then flips all spins of this cluster. A typical example is shown in Fig. 5. In principle, one could also here choose a value +1 or -1 at random, but then nothing at all would be changed if one hits the current value of the spins. Here a sweep consists of $V /\langle|C|\rangle$ single cluster steps, where $\langle|C|\rangle$ denotes the average cluster size. With this definition autocorrelation times are directly comparable with results from the Metropolis or Swendsen-Wang algorithm. Apart from being somewhat easier to program, the single-cluster variant is usually more efficient than the multiple-cluster algorithm, especially in 3D [24]. The reason is that with the single-cluster method, on average, larger clusters are flipped. A rigorous lower bound on $z$ for the multiple-cluster algorithms has been given in [25].

The performance of the two cluster and the Metropolis algorithm for the Ising model is compared in Table 1.

\subsubsection{Embedded cluster}

While it is straightforward to generalize the derivation (22)-(25) to $q$-state Potts models [22], for the $\mathrm{O}(n)$ spin models with $n \geq 2$, one needs a new strategy [23,30-34]. Here the basic idea [23,30] is to isolate Ising degrees of freedom by projecting $\vec{\sigma}_{i}$ onto a randomly chosen unit vector $\vec{r}$,

$$
\vec{\sigma}_{i}=\vec{\sigma}_{i}^{\|}+\vec{\sigma}_{i}^{\perp}, \quad \vec{\sigma}_{i}^{\|}=\epsilon\left|\vec{\sigma}_{i} \cdot \vec{r}\right| \vec{r}, \epsilon=\operatorname{sign}\left(\vec{\sigma}_{i} \cdot \vec{r}\right)
$$

If this is inserted into the original Hamiltonian one ends up with an effective Hamiltonian 
Table 1

Dynamic critical exponents $z$ for the Ising model $\left(\tau \propto L^{z}\right)$

\begin{tabular}{|c|c|c|c|c|}
\hline Algorithm & $D=2$ & $D=3$ & Observable & Reference \\
\hline Metropolis & 2.125 & 2.03 & & \\
\hline \multirow[t]{5}{*}{ Swendsen-Wang cluster } & $0.35(1)$ & $0.75(1)$ & $z_{E, \exp }$ & {$[22]$} \\
\hline & $0.27(2)$ & $0.50(3)$ & $z_{E, \text { int }}$ & {$[26]$} \\
\hline & $0.20(2)$ & $0.50(3)$ & $z_{\chi, \text { int }}$ & [26] \\
\hline & $0(\log L)$ & - & $z_{M, \exp }$ & [27] \\
\hline & $0.25(5)$ & - & $z_{M, \mathrm{rel}}$ & {$[28]$} \\
\hline \multirow[t]{3}{*}{ Wolff cluster } & $0.26(2)$ & $0.28(2)$ & $z_{E, \text { int }}$ & {$[26]$} \\
\hline & $0.13(2)$ & $0.14(2)$ & $z_{\chi, \text { int }}$ & [26] \\
\hline & $0.25(5)$ & $0.3(1)$ & $z_{E, \text { rel }}$ & [29] \\
\hline
\end{tabular}

$$
H=-\sum_{\langle i j\rangle} J_{i j} \epsilon_{i} \epsilon_{j}+\text { const. }
$$

with positive random couplings,

$$
J_{i j}=J\left|\vec{\sigma}_{i} \cdot \vec{r}\right|\left|\vec{\sigma}_{j} \cdot \vec{r}\right| \geq 0
$$

whose Ising degrees of freedom $\epsilon_{i}$ can be updated with a cluster algorithm as described above.

Also for $\mathrm{O}(n)$ spin models with $n \geq 2$ both types of cluster algorithms reduce critical slowing down drastically. As expected in three dimensions the Wolff cluster algorithm performs again better than the Swendsen-Wang algorithm. For the 3D XY model the estimates of the dynamical critical exponents are $z_{E \text {,int }} \approx 0.46$ and $z_{\chi, \text { int }} \approx 0.31$ for SW [35] and $z_{E \text {,int }} \approx 0.25$ and $z_{\chi, \text { int }} \approx 0$ for SC [36]. Compared with the Metropolis algorithm, factors of up to 10000 in CPU time have been saved in realistic simulations $[37,38]$.

\subsubsection{Improved estimators}

A further merit of cluster algorithms is that they lead quite naturally to alternative estimators with the same expectation but different variance [31,32,39]. For the correlation function $G\left(\vec{x}_{i}-\vec{x}_{j}\right) \equiv\left\langle\vec{\sigma}_{i} \cdot \vec{\sigma}_{j}\right\rangle$ in the high-temperature phase and the single-cluster formulation it reads [31]

$$
\hat{G}\left(\vec{x}_{i}-\vec{x}_{j}\right)=n \frac{V}{|C|} \vec{r} \cdot \vec{\sigma}_{i} \vec{r} \cdot \vec{\sigma}_{j} \Theta_{C}\left(\vec{x}_{i}\right) \Theta_{C}\left(\vec{x}_{j}\right)
$$

where $|C|$ denotes the sizes of the cluster and $\Theta_{C}(\vec{x})$ is its characteristic function $(=1$ if $\vec{x} \in C$ and 0 otherwise). For the susceptibility this implies

$$
\hat{\chi}^{\prime} / \beta=\frac{n}{|C|}\left(\sum_{i \in C} \vec{r} \cdot \vec{\sigma}_{i}\right)^{2}
$$

which simplifies for the Ising model $(n=1)$ to $\hat{\chi}^{\prime} / \beta=|C|$, i.e., the average cluster size in the singlecluster formulation is an estimator of the susceptibility. By using the estimators Eqs. (29) and (30) a 
Table 2

Critical coupling and critical exponents of the 3D classical Heisenberg $(n=3)$ model

\begin{tabular}{|c|c|c|c|c|}
\hline Method & $\beta_{\mathrm{c}}$ & $\nu$ & $\gamma$ & Reference \\
\hline$g$-Expansion & - & $0.705(3)$ & $1.386(4)$ & {$[43]$} \\
\hline$\epsilon$-Expansion & - & $0.710(7)$ & $1.390(10)$ & {$[44]$} \\
\hline MC FSS & $0.6929(1)$ & $0.706(9)$ & $1.390(23)$ & {$[45]$} \\
\hline MC FSS & $0.6930(2)$ & $0.73(4)$ & - & {$[46]$} \\
\hline MC FSS & $0.6930(1)$ & $0.704(6)$ & $1.389(14)$ & {$[40,41]$} \\
\hline $\mathrm{MC} T>T_{\mathrm{c}}$ & 0.69288 & $0.698(2)$ & $1.391(3)$ & {$[41]$} \\
\hline MC FSS & $0.693035(37)$ & $0.7036(23)$ & $1.3896(70)$ & {$[47]$} \\
\hline MC FSS & $0.693002(12)$ & $0.7128(14)$ & $1.399(2)$ & {$[48]$} \\
\hline MC FSS & - & $0.699(3)$ & - & [49] \\
\hline Series (14 terms) & $0.6929(1)$ & $0.712(10)$ & $1.400(10)$ & {$[50]$} \\
\hline Series (19 terms) & $0.69302(7)$ & $0.715(3)$ & $1.403(6)$ & {$[51]$} \\
\hline Series (21 terms) & $0.69303(3)$ & $0.715(2)$ & $1.404(3)$ & {$[52]$} \\
\hline
\end{tabular}

significant reduction of variance can be expected outside the FSS region where the average cluster size is small compared with the size of the system [31,36,40,41]. A variant of Eq. (29) has recently been discussed in [42].

As an example of what can be achieved by these nonlocal MC algorithms recent estimates of the static critical exponents of the 3D Heisenberg model are compared in Table 2 with results from field theory and high-temperature series expansions.

\subsubsection{Further generalizations}

The derivation (22)-(24) generalizes almost trivially to Potts models [53] with Hamiltonian $H=-J \sum_{\langle i j\rangle} \delta_{\sigma_{i} \sigma_{j}}$, and $\sigma_{i}=1, \ldots, q[22,54,55]$. Also next-nearest neighbor interactions [56] and even long-range interactions including the mean-field limit [57] can be treated in an analogous way. For external magnetic fields, several related formulations have been tested [58]. Microcanonical cluster variants are discussed in [59], and combinations of cluster-update algorithms with multicanoncial simulations of first-order phase transitions have been proposed and tested in [60-62].

Randomly diluted ferromagnetic systems such as the 2D random bond Ising [63,64] and Potts [65] model or the 3D site diluted Ising model [66] pose no new problems, and simulations on random lattices [67] or graphs [68] and fluctuating Regge triangulations [69] of quantum gravity are also straightforward. For systems with competing ferro- and antiferromagnetic interactions and frustrations, on the other hand, it is not guaranteed that a straightforward cluster formulation leads to improvements even though this would be a valid algorithm. Examples are Edwards-Anderson Ising spin glasses in 2D [70] and 3D [71] (see also [72]). A successful algorithm could be derived for the fully frustrated model [73]. A comprehensive discussion of the general construction principle is given in [74,75]. Among others this has led to cluster methods for the Ashkin-Teller model [76,77]. Fast cluster algorithms could also be derived for antiferromagnetic Potts models on square [78] and triangular [79] lattices.

Generalizations of the embedding idea have been investigated for the $\Phi^{4}$ lattice field theory [80] and also for quite general symmetry groups [81]. For asymmetric models interpolating between $\mathrm{O}(n)$ and $\mathrm{O}(n+1)$ symmetry see [82]. Along similar lines, efficient cluster algorithms have been derived for the $Z_{2}$ lattice gauge theory [83], solid-on-solid models [84] and fluctuating interfaces [85]. 
Cluster algorithms can also be formulated for vertex models [86] and for some quantum statistical sytems [87-89].

\subsection{Multigrid algorithms}

A different class of nonlocal update algorithms is based on multigrid techniques which have proven to be a very successful mathematical tool for the numerical solution of partial differential equations. In this context there exists a vast literature at text book level [90], and quite a few properties of deterministic multigrid schemes are known exactly. The basic idea is to deal with many length scales at a time. About a decade ago this multilevel approach was also adapted to the stochastic problems of MC sampling [91-100]. In contrast to cluster-update algorithms, where the average cluster size is determined in course of the simulation by the system under study itself and which are therefore self-adaptive, for multigrid algorithms the different length scales are explicit input parameters.

\subsubsection{Unigrid and multigrid formulations}

In the unigrid formulation one works with the original $D$-dimensional lattice and usually proposes collective moves for blocks of neighboring field variables $\phi_{i}$ of size $1,2^{D}, 4^{D}, 8^{D}, \ldots, 2^{n D}=V$. The proposed moves of fixed shape but variable amplitude are then accepted or rejected according to the energy change, similar to the Metropolis algorithm. The simplest choice are the above piecewise constant collective excitations (blocks), but in principle other "smoother" shapes could also be considered (e.g., pyramids). Another important ingredient is the sequence in which the various block sizes are selected in a multigrid cycle, the analog of a sweep.

In the equivalent recursive multigrid formulation the blocks of size $2^{(n-k) D}$ are represented by auxiliary variables $\phi_{i}^{(k)}$ on coarse-grained lattices - the multigrids $\Xi^{(k)}$ of size $2^{k D}, k=n, \ldots, 0$. Here the shape of the collective moves is formally controlled by an operator $\mathscr{P}$ which interpolates $\phi_{i}^{(k)}$ back to the next finer grid $\Xi^{(k+1)}$, and the acceptance of the proposed moves is governed by a coarse-grid Hamiltonian, $H^{(k)}\left(\phi_{i}^{(k)}\right)=H^{(k+1)}\left(\phi_{i}^{(k+1)}+\mathscr{P} \phi_{i}^{(k)}\right), H^{(n)}=H$, which is defined recursively by freezing the field variables $\phi_{i}^{(k+1)}$.

For practical implementation, it is important that the $H^{(k)}$ are form-invariant under the coarsening. The update on level $\Xi^{(k)}$ thus consists of:

1. $n_{1}$ presweeps using any valid local update scheme with Hamiltonian $H^{(k)}$;

2. calculating the Hamiltonian for the next coarser grid $\Xi^{(k-1)}$ (whose parameters depend on the current configuration on grid $\Xi^{(k)}$ and initializing the variables on grid $\Xi^{(k-1)}$ to zero;

3. updating the field variables $\phi_{i}^{(k-1)}$ with the multigrid scheme $\gamma_{k-1}$ times;

4. interpolating the variables of grid $\Xi^{(k-1)}$ back to grid $\Xi^{(k)}$;

5. $n_{2}$ postsweeps using the local update algorithm with Hamiltonian $H^{(k)}$.

On the coarsest grid $\Xi^{(0)}$, of course, one only performs steps 1 and 5 .

The two viewpoints are mathematically equivalent, but the multigrid formulation leads automatically to a more efficient implementation if the functional form of the Hamiltonian remains invariant under the coarsening prescription (similar to FFT) and, due to its recursive structure, makes the definition of level sequences determined by the parameters $\gamma_{k}$ more transparent. For $\gamma_{k} \equiv 1$ (V-cycle) every grid is given the same weight, while for $\gamma_{k} \equiv 2$ (W-cycle) the coarser grids are updated more 

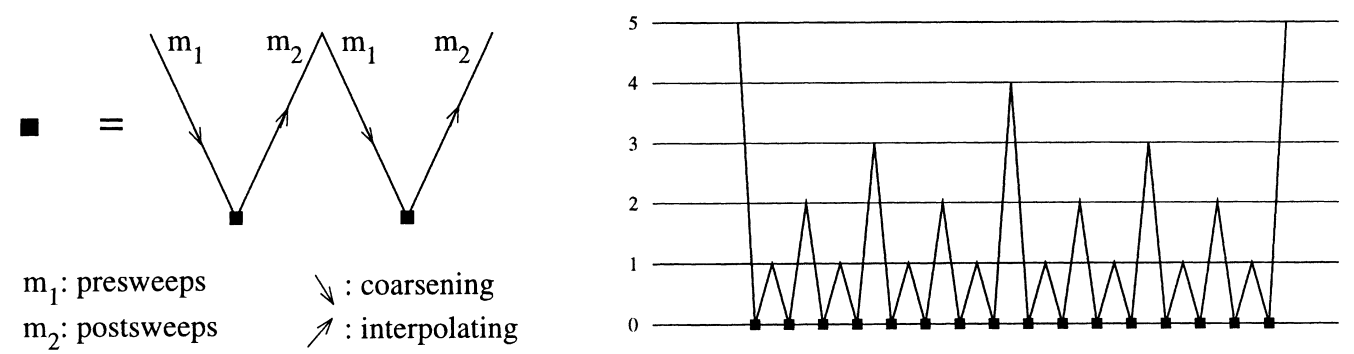

Fig. 6. The recursive definition of the W-cycle and the expanded W-cycle for $L=32$.

frequently. The cycle names derive from their graphical representation which resemble the letters $\mathrm{V}$ and W. (see Fig. 6).

Besides the choice of the cycle, the interpolation operator $\mathscr{P}$ is the other important parameter of multigrid update schemes [101,102]. By making use of the close relationship with deterministic multigrid techniques for the numerical solution of partial differential equations [90], it can be shown that for Gaussian (free) fields and piecewise constant interpolation, critical slowing down is completely eliminated for the W-cycle [92]. For the V-cycle no analytic results are known, but numerical evidence suggests that $z=1$ in this case. For higher order "smooth" interpolations (e.g., the "pyramids" in linear interpolation), on the other hand, also the V-cycle eliminates critical slowing down. In all interesting physical applications the fields are non-Gaussian, but empirically we know that these exact results can be taken at least as a useful guideline.

When judging the efficiency of various combinations of interpolation operators and multigrid cycles it is important to take into account the numerical work spent in one cycle, the analog of a sweep. With the definition $f=$ work(cycle)/work(Metropolis sweep) one finds for the V-cycle in all dimensions $f=$ constant in the recursive multigrid and $f \propto \log L$ in the unigrid formulation. For the W-cycle the recursive formulation gives $f \propto \log L$ in one dimension and $f=$ constant in higher dimensions, and the unigrid formulation always leads to $f \propto L$. For piecewise constant interpolation it is often possible to exploit the advantages of the recursive formulation, so that the implementation of a W-cycle is almost as fast as that of a V-cycle. For higher order interpolations, on the other hand, the recursive formulation is usually not feasible and one resorts to the unigrid formulation, where the implementation of the Wcycle is slower than that of the $\mathrm{V}$-cycle by a factor of $L / \log L$. This, however, is not a real drawback since, for smooth interpolations, the dynamical behavior of the multigrid approach is better and also the V-cycle drastically reduces critical slowing down. From these estimates it should be clear that a comparison of the performance of a "recursive W-cycle with piecewise constant interpolation" and a "unigrid V-cycle with smooth interpolation" is somewhat subtle, and there has been indeed much debate about this point in the literature.

\subsubsection{Applications to path-integral Monte Carlo}

As an example we shall illustrate the use of multigrid algorithms in the path-integral formulation of quantum statistics [103]. In this formulation the partition function $Z=\operatorname{Tr} \exp (-\beta \hat{H})$ of a 


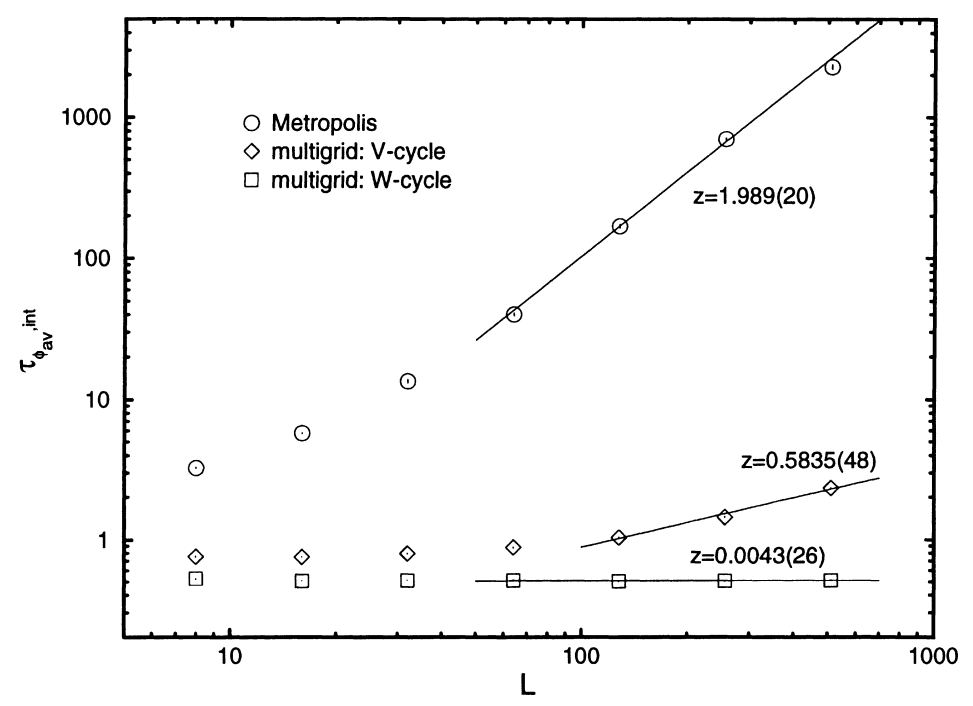

Fig. 7. Integrated autocorrelation times for $\phi_{\mathrm{av}}$.

$D$-dimensional many-particle quantum system with Hamiltonian $\hat{H}=\sum_{i=1}^{N} \hat{p}_{i}^{2} / 2 m_{i}+V\left(\left\{\hat{\phi}_{i}\right\}\right)$ (the "hats" denote operators) is written as $Z=\lim _{L \rightarrow \infty} Z_{L}$ with $(\hbar=1)$ :

$$
Z_{L}=\prod_{i=1}^{N} \prod_{k=1}^{L} \int \frac{\mathrm{d} \phi_{i, k}}{\sqrt{2 \pi \epsilon}} \exp \left[-\epsilon \sum_{i=1}^{N} \sum_{k=1}^{L}\left(\frac{1}{2}\left(\frac{\phi_{i, k+1}-\phi_{i, k}}{\epsilon}\right)^{2}+V\left(\left\{\phi_{i, k}\right\}\right)\right)\right]
$$

where the $\phi_{i, k}$ are ordinary real numbers, $\epsilon L=\beta$, and $\phi_{i, L+1}=\phi_{i, 1}$ for all $i$. This looks like a $(D+1)$ dimensional classical partition function with continuous degrees of freedom and can hence be simulated with any valid update algorithm, in particular with multigrid techniques. In [96] we first considered the simpler one-particle case with an anharmonic potential $V(\phi)=\frac{1}{2} \phi^{2}+\phi^{4}$ and measured the autocorrelation times of the average field $\phi_{\mathrm{av}}=(1 / L) \sum_{k=1}^{L} \phi_{k}$ as a function of the temporal grid size $L$. The results for $\beta=10$ are shown in Fig. 7. We see that as expected the local Metropolis algorithm is again governed by a dynamical exponent $z \approx 2$. The multigrid cycles with piecewise constant interpolation, on the other hand, lead to a significant reduction of autocorrelations. In particular for the $\mathrm{W}$-cycle the slowing down seems to be completely eliminated. The same pattern was observed for a double-well potential $V(\phi)=\frac{1}{2} \phi^{2}+0.04 \phi^{4}$ [96], and also for quantum chains [104].

It is worth pointing out that for path-integral applications one could also employ the very successful staging algorithm [105] which is close in spirit but quite different technically. For a careful comparison of multigrid and staging algorithms see [106].

\subsubsection{Applications to spin models and field theory}

In the past few years there have been numerous applications of the multigrid MC approach to lattice spin and field theoretic models. Examples are studies of the 2D XY model [107], 2D Heisenberg model [108], the $\mathrm{O}(4)$ symmetric spin model in $1 \mathrm{D}$ [109] and 2D [110], and the 2D $\mathrm{CP}^{3}$ model [111]. More recently the $2 \mathrm{D} \mathrm{O}(n)$ symmetric models [112] and the SU(3) principal chiral model [113] have been studied by employing the so-called XY-embedding. For the $2 \mathrm{D} \mathrm{O}(n)$ symmetric models with $n=3,4$ 
and 8 , the dynamical critical exponent turned out to be quite small, $z \approx 0.50-0.70$, but it is definitely not zero [112]. In the cases where cluster update algorithms are also applicable, these are thus usually more efficient than multigrid simulations. But there are also other cases, such as $\sigma$ models taking values in the group $\mathrm{SU}(n)$ with $n \geq 3$, where embedding-type cluster algorithms are not expected to work [81], and multigrid MC is the only known nonlocal algorithm (except perhaps Fourier acceleration) that has a chance of achieving $z \ll 2$. Another application of multigrid methods, where cluster algorithms do not work, is 4D U(1) lattice gauge theory [114]. Attempts to adapt the method to non-abelian lattice gauge theories and further references can be found in [101]. The combination of multigrid update schemes with multicanonical simulations is discussed in [115], and investigations of optimization strategies employing a careful scheduling of measurements taken at the coarse grids have been reported in [116].

\section{Concluding remarks}

The intention of this paper was to give an elementary introduction to the basic concepts of modern MC simulations using nonlocal update algorithms and to illustrate their usefulness by applications to a few typical models.

By comparing the various MC estimates collected in Table 2 with results from field theory and series expansions it is fair to conclude that for $\mathrm{O}(n)$ spin models modern $\mathrm{MC}$ cluster update techniques give more accurate results than series expansion analyses which are, however, also constantly improving. The recently derived critical exponents are in fact competitive in accuracy with estimates obtained with the best and very elaborate methods of field theory.

For $\mathrm{O}(n)$ spin models multigrid techniques are usually less efficient than cluster algorithms. They are very successful, however, in path-integral MC simulation where no cluster updates are available. There are also many other models where only multigrid methods have a real chance of reducing critical slowing down.

To some extent the range of applicability of the two nonlocal update algorithms is thus complimentary, and it is this fact which makes pursuing investigations of both approaches worthwhile.

\section{Acknowledgements}

It is a pleasure to thank Joan Adler, Achi Brandt, Malcolm Carroll, Christian Holm, Desmond Johnston, Stefan Kappler, Tilman Sauer, Sergei Shmulyian and Ramon Villanova for fruitful collaborations, and Kurt Binder and David Landau for helpful discussions. Finally, I gratefully acknowledge support from the Deutsche Forschungsgemeinschaft (DFG) through a Heisenberg Fellowship.

\section{References}

[1] H.E. Stanley, Introduction to Phase Transitions and Critical Phenomena, Oxford Press, Oxford, 1979; current research topics are reviewed in: C. Domb, J.L. Lebowitz (Eds.), Phase Transitions and Critical Phenomena, Academic Press, London. 
[2] J.D. Gunton, M.S. Miguel, P.S. Sahni, in: C. Domb, J.L. Lebowitz (Eds.), Phase Transitions and Critical Phenomena, vol. 8, Academic Press, New York, 1983, p. 269.

[3] K. Binder, Rep. Prog. Phys. 50 (1987) 783.

[4] H.J. Herrmann, W. Janke, F. Karsch (Eds.), Dynamics of First Order Phase Transitions, World Scientific, Singapore, 1992.

[5] W. Janke, in: D.P. Landau, K.K. Mon, H.-B. Schüttler (Eds.), Computer Simulations in Condensed Matter Physics, vol. VII, Springer, Berlin, 1994, p. 29.

[6] H. Kleinert, Gauge Fields in Condensed Matter, Vol II, World Scientific, Singapore, 1989.

[7] W. Janke, H. Kleinert, Phys. Rev. B 33 (1986) 6346.

[8] K. Binder, in: K. Binder (Ed.), Monte Carlo Methods in Statistical Physics, Springer, Berlin, 1979, p. 1.

[9] M.E. Barber, in: C. Domb, J.L. Lebowitz (Eds.), Phase Transitions and Critical Phenomena, vol. 8, Academic Press, New York, 1983, p. 146.

[10] V. Privman (Ed.), Finite-Size Scaling and Numerical Simulations of Statistical Systems, World Scientific, Singapore, 1990.

[11] K. Binder, in: H. Gausterer, C.B. Lang (Eds.), Computational Methods in Field Theory, Schladming Lecture Notes, Springer, Berlin, 1992, p. 59.

[12] W. Lenz, Phys. Z. 21 (1920) 613; E. Ising, Z. Phys. 31 (1925) 253.

[13] J.M. Hammersley, D.C. Handscomb, Monte Carlo Methods, Chapman and Hall, London, 1964.

[14] K. Binder, D.W. Heermann, Monte Carlo Simulations in Statistical Physics: An Introduction, Springer, Berlin, 1988.

[15] D.W. Heermann, Computer Simulation Methods in Theoretical Physics, 2nd edn., Springer, Berlin, 1990.

[16] K. Binder (Ed.), The Monte Carlo Method in Condensed Matter Physics, Springer, Berlin, 1992.

[17] N. Metropolis, A. Rosenbluth, M. Rosenbluth, A. Teller, E. Teller, J. Chem. Phys. 21 (1953) 1087.

[18] N. Madras, A.D. Sokal, J. Stat. Phys. 50 (1988) 109.

[19] P.W. Kasteleyn, C.M. Fortuin, J. Phys. Soc. Jpn. 26 (Suppl.) (1969) 11; C.M. Fortuin, P.W. Kasteleyn, Physica 57 (1972) 536; C.M. Fortuin, Physica 58 (1972) 393; ibid. 59 (1972) 545.

[20] A.N. Burkitt, D.W. Heermann, Comp. Phys. Commun. 54 (1989) 210; C.F. Baillie, P.D. Coddington, Phys. Rev. B 43 (1991) 10617; M. Flanigan, P. Tamayo, Int. J. Mod. Phys. C 3 (1992) 1235; J. Kertesz, D. Stauffer, Int. J. Mod. Phys. C 3 (1992) 1275; M. Bauernfeind, R. Hackl, H.-G. Matuttis, J.M. Singer, Th. Husslein, I. Morgenstern, Physica A 212 (1994) 277; G.T. Barkema, T. MacFarland, Phys. Rev. E 50 (1994) 1623; M. Flanigan, P. Tamayo, Physica A 215 (1995) 461.

[21] H. Mino, Comp. Phys. Commun. 66 (1991) 25; H.G. Evertz, Nucl. Phys. B (Proc. Suppl.) 26 (1992) 620.

[22] R.H. Swendsen, J.-S. Wang, Phys. Rev. Lett. 58 (1987) 86.

[23] U. Wolff, Phys. Rev. Lett. 62 (1989) 361.

[24] A.D. Sokal, Nucl. Phys. B (Proc. Suppl.) 20 (1991) 55.

[25] X.-L. Li, A.D. Sokal, Phys. Rev. Lett. 63 (1989) 827; ibid. 671482 (1991).

[26] U. Wolff, Phys. Lett. A 228 (1989) 379.

[27] D.W. Heermann, A. N Burkitt, Physica A 162 (1990) 210.

[28] P. Tamayo, Physica A 201 (1993) 543.

[29] N. Ito, G.A. Kohring, Physica A 201 (1993) 547.

[30] U. Wolff, Nucl. Phys. B 322 (1989) 759.

[31] U. Wolff, Nucl. Phys. B 334 (1990) 581.

[32] M. Hasenbusch, Nucl. Phys. B 333 (1990) 581.

[33] R.G. Edwards, A.D. Sokal, Phys. Rev. D 38 (1988) 2009; ibid. D 40 (1989) 1374.

[34] F. Niedermayer, Phys. Rev. Lett. 61 (1988) 2026.

[35] M. Hasenbusch, S. Meyer, Phys. Lett. B 241 (1990) 238.

[36] W. Janke, Phys. Lett. A 148 (1990) 306.

[37] C.F. Baillie, Int. J. Mod. Phys. C 1 (1990) 91.

[38] R.H. Swendsen, J.-S. Wang, A.M. Ferrenberg in: K. Binder (Ed.), The Monte Carlo Method in Condensed Matter Physics, Springer, Berlin, 1992, p. 75.

[39] F. Niedermayer, Phys. Lett. B 237 (1990) 473.

[40] C. Holm, W. Janke, Phys. Lett. A 173 (1993) 8.

[41] C. Holm, W. Janke, Phys. Rev. B 48 (1993) 936. 
[42] W. Janke, S. Kappler, Phys. Rev. E 56 (1997) 1414.

[43] J.C. Le Guillou, J. Zinn-Justin, Phys. Rev. Lett. 39 (1977) 95; Phys Rev. B 21 (1980) 3976.

[44] J.C. Le Guillou, J. Zinn-Justin, J. Phys. Lett. 46 (1985) L137.

[45] P. Peczak, A.M. Ferrenberg, D.P. Landau, Phys. Rev. B 43 (1991) 6087.

[46] I. Dimitrović, P. Hasenfratz, J. Nager, F. Niedermayer, Nucl. Phys. B 350 (1991) 893.

[47] K. Chen, A.M. Ferrenberg, D.P. Landau, Phys. Rev. B 48 (1993) 3249.

[48] H.G. Ballesteros, L.A. Fernández, V. Martín-Mayor, A. Muñoz Sudupe, Phys. Lett. B 387 (1996) 125.

[49] C. Holm, W. Janke, J. Phys. A 27 (1994) 2553; Phys. Rev. Lett. 78 (1997) 2265.

[50] J. Adler, C. Holm, W. Janke, Physica A 201 (1993) 581.

[51] P. Butera, M. Comi, Phys. Rev. B 52 (1995) 6185.

[52] P. Butera, M. Comi, Phys. Rev. B 56 (1997) 8272.

[53] R.B. Potts, Proc. Camb. Phil. Soc. 48 (1952) 106; F.Y. Wu, Rev. Mod. Phys. 54 (1982) 235; ibid. 55 (1983) $315 E$.

[54] W. Janke, S. Kappler, Phys. Lett. A 197 (1995) 227; Europhys. Lett. 31 (1995) 345.

[55] S. Kappler, Ph.D. Thesis, Johannes Gutenberg-Universität Mainz, 1995.

[56] E. Marinari, R. Marra, Nucl. Phys. B 342 (1990) 737.

[57] E. Luijten, H.W.J. Blöte, Int. J. Mod. Phys. C 6 (1995) 359; E. Luijten, H.W.J. Blöte, K. Binder, Phys. Rev. E 54 (1996) 4626; E. Luijten, H.W.J. Blöte, Delft Preprint, 1997.

[58] J.-S. Wang, Physica A 161 (1989) 249; P.G. Lauwers, V. Rittenberg, Phys. Lett. B 233 (1989) 197; V.S. Dotsenko, W. Selke, A.L. Talapov, Physica A 170 (1991) 278.

[59] M. Creutz, Phys. Rev. Lett. 69 (1992) 1002.

[60] K. Rummukainen, Nucl. Phys. B 390 (1993) 621.

[61] W. Janke, S. Kappler, Phys. Rev. Lett. 74 (1995) 212; M.S. Carroll, W. Janke, S. Kappler, to appear in J. Stat. Phys. 90 (1998).

[62] W. Janke, in: F. Karsch, B. Monien, H. Satz (Eds.) Multiscale Phenomena and their Simulation, World Scientific, Singapore, 1997, p. 147.

[63] J.-S. Wang, W. Selke, Vl.S. Dotsenko, V.B. Andreichenko, Europhys. Lett. 11 (1990) 301; Physica A 164 (1990) 221; V.B. Andreichenko, Vl.S. Dotsenko, W. Selke, J.-S. Wang, Nucl. Phys. B 344 (1990) 531.

[64] A.L. Talapov, L.N. Shchur, Europhys. Lett. 27 (1994) 193; J. Cond. Matter 6 (1994) 8295.

[65] S. Chen, A.M. Ferrenberg, D.P. Landau, Phys. Rev. Lett. 69 (1992) 1213; Phys. Rev. E 52 (1995) 1377.

[66] J.-S. Wang, D. Chowdhury, J. Phys. (Paris) 50 (1989) 2905; J.-S. Wang, M. Wöhlert, H. Mühlenbein, D. Chowdhury, Physica A 166 (1990) 173.

[67] W. Janke, M. Katoot, R. Villanova, Phys. Lett. B 315 (1993) 412; Phys. Rev. B 49 (1994) 9644; W. Janke, R. Villanova, Phys. Lett. A 209 (1995) 179.

[68] C.F. Baillie, W. Janke, D.A. Johnston, Phys. Lett. B 388 (1996) 14.

[69] C. Holm, W. Janke, Phys. Lett. B 335 (1994) 143; ibid. B 375 (1996) 69.

[70] V. Cataudella, Physica A 183 (1991) 249.

[71] L. de Arcangelis, A. Coniglio, F. Peruggi, Europhys. Lett. 14 (1991) 515.

[72] P.W. Leung, C.L. Henley, Phys. Rev. B 43 (1991) 752.

[73] D. Kandel, R. Ben-Av, E. Domany, Phys. Rev. Lett. 65 (1990) 941; Phys Rev. B 45 (1992) 4700.

[74] D. Kandel, E. Domany, Phys. Rev. B 43 (1991) 8539.

[75] V. Cataudella, G. Franzes, M. Nicodemi, A. Scala, A. Coniglio, Phys. Rev. Lett. 72 (1994) 1541.

[76] S. Wiseman, E. Domany, Phys. Rev. E 48 (1993) 4080.

[77] J. Salas, A.D. Sokal, J. Stat. Phys. 86 (1996) 297.

[78] Wang, J.-S. Swendsen, R.H. Kotecký, R. Phys. Rev. Lett. 63 (1989) 109; Phys. Rev. B 42 (1990) 2465; M. Lubin, A.D. Sokal, Phys. Rev. Lett. 71 (1993) 1778.

[79] J. Adler, A. Brandt, W. Janke, S. Shmulyian, J. Phys. A 28 (1995) 5117.

[80] R.C. Brower, P. Tamayo, Phys. Rev. Lett. 62 (1989) 1087; Ch. Frick, K. Jansen, P. Seuferling, Phys. Rev. Lett. 63 (1989) 2613; R.C. Brower, N.A. Gross, K.J.M. Moriarty, J. Comp. Phys. 95 (1991) 167.

[81] S. Caracciolo, R.G. Edwards, A. Pelissetto, A.D. Sokal, Nucl. Phys. B 403 (1993) 475.

[82] M. Klomfass, U.M. Heller, H. Flyvbjerg, Phys. Lett. B 258 (1991) 386; Nucl. Phys. B 360 (1991) 264.

[83] R. Ben-Av, D. Kandel, E. Katznelson, P.G. Lauwers, S. Solomon, J. Stat. Phys. 58 (1990) 125.

[84] H.G. Evertz, M. Marcu, K. Pinn, S. Solomon, Phys. Lett. B 254 (1991) 185. 
[85] M. Hasenbusch, S. Meyer, Phys. Rev. Lett. 66 (1991) 530; M. Hasenbusch, K. Pinn, Physica A 192 (1993) 342.

[86] H.G. Evertz, G. Lana, M. Marcu, Phys. Rev. Lett. 70 (1993) 875.

[87] U.-J. Wiese, H.-P. Ying, Z. Phys. B 93 (1994) 147.

[88] B.B. Beard, U.-J. Wiese, Nucl. Phys. B (Proc. Suppl.) 53 (1997) 838.

[89] N. Kawashima, J.E. Gubernatis, H.G. Evertz, Phys. Rev. B 50 (1994) 136.

[90] W. Hackbusch, Multi-Grid Methods and Applications, Springer, Berlin, 1985; W.L. Briggs, A Multigrid Tutorial, SIAM, Philadelphia, 1987; S.F. McCormick (Ed.), Multigrid Methods: Theory, Applications, and Supercomputing, Dekker, New York, 1988.

[91] H. Meyer-Ortmanns, Z. Physik C 27 (1985) 553.

[92] J. Goodman, A.D. Sokal, Phys. Rev. Lett. 56 (1986) 1015; Phys. Rev. D 40 (1989) 2035.

[93] G. Mack, in: G.'t Hooft et al. (Eds.), Nonperturbative Quantum Field Theory, Cargèse lectures, 1987, Plenum, New York, 1988, p. 309; G. Mack, S. Meyer, Nucl. Phys. B (Proc. Suppl.) 17 (1990) 293.

[94] D. Kandel, E. Domany, D. Ron, A. Brandt, E. Loh, Jr., Phys. Rev. Lett. 60 (1988) 1591; D. Kandel, E. Domany, A. Brandt, Phys. Rev. B 40 (1989) 330.

[95] A. Brandt, Nucl. Phys. B (Proc. Suppl.) 26 (1992) 137.

[96] W. Janke, T. Sauer, Chem. Phys. Lett. 201 (1993) 499.

[97] W. Janke, T. Sauer, in: H. Grabert et al. (Eds.) Path Integrals from meV to MeV, World Scientific, Singapore, 1993, p. 17.

[98] T. Sauer, Ph.D. Thesis, Freie Universität Berlin, 1994.

[99] A.D. Sokal, Monte Carlo Methods in Statistical Mechanics: Foundations and New Algorithms, Cours de Troisième Cycle de la Physique en Suisse Romande, Lausanne, 1989.

[100] A.D. Sokal, Bosonic algorithms, in: M. Creutz (Ed.), Quantum Fields on the Computer, World Scientific, Singapore, 1992, p. 211.

[101] M. Grabenstein, Ph.D. Thesis, Hamburg University, 1994, DESY preprint 94-007, hep-lat/9401024.

[102] M. Grabenstein, K. Pinn, Phys. Rev. D45, 4372 (1992); Nucl. Phys. B (Proc. Suppl.) 30 (1993) 265; J. Stat. Phys. 71 (1993) 607.

[103] H. Kleinert, Path Integrals in Quantum Mechanics, Statistics and Polymer Physics, 2nd edn., World Scientific, Singapore, 1995.

[104] W. Janke, T. Sauer, Phys. Lett. A 197 (1995) 335.

[105] E.L. Pollock, D.M. Ceperley, Phys. Rev. B 30 (1984) 2555; M. Sprik, M.L. Klein, D. Chandler, Phys. Rev. B 31 (1985) 4234; ibid. B 32 (1985) 545; M.E. Tuckerman, B.J. Berne, G.J. Martyna, M.L. Klein, J. Chem. Phys. 99 (1993) 2796.

[106] W. Janke, T. Sauer, Chem. Phys. Lett. 263 (1996) 448; and J. Chem. Phys. 107 (1997) 5821.

[107] R.G. Edwards, J. Goodman, A.D. Sokal, Nucl. Phys. B 354 (1991) 289; A. Hulsebos, J. Smit, J.C. Vink, Nucl. Phys. B 356 (1991) 775.

[108] M. Hasenbusch, S. Meyer, Nucl. Phys. B (Proc. Suppl.) 20 (1991) 110.

[109] T. Mendes, A.D. Sokal, Phys. Rev. D 53 (1996) 3438.

[110] R.G. Edwards, S.J. Ferreira, J. Goodman, A.D. Sokal, Nucl. Phys. B 380 (1992) 621.

[111] M. Hasenbusch, S. Meyer, Phys. Rev. D 45 (1992) R4376.

[112] T. Mendes, A. Pelissetto, A.D. Sokal, Nucl. Phys. B 477 (1996) 203.

[113] G. Mana, A. Pelissetto, A.D. Sokal, Phys. Rev. D 55 (1997) 3674.

[114] M.L. Laursen, J.C. Vink, Nucl. Phys. B 401 (1993) 745.

[115] W. Janke, T. Sauer, Phys. Rev. E 49 (1994) 3475; J. Stat. Phys. 78 (1995) 759.

[116] A. Brandt, M. Galun, D. Ron, J. Stat. Phys. 74 (1994) 313. 